
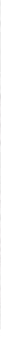

Gerenciamento de Cidades

\title{
Energia solar como solução energética sustentável em cidade compactas
}

Solar energy as a sustainable energy solution in compact cities

Energía solar como solución energética sostenible en ciudad compacta

\author{
Clerismar Fernandes da Silva \\ Mestranda em Eng. Urbana, PEU/POLI/UFRJ, Brasil \\ clerys.eel@poli.ufrj.br
}

Patrícia Regina Chaves Drach

Professora Doutora, DAU/ESDI/UERJ, Brasil Professora Doutora, PROURB/UFRJ, Brasil

Pesquisadora, PEU/UFRJ, Brasil patricia.drach@gmai.com

Gisele Silva Barbosa

Professora Doutora, PEU/POLI/UFRJ, Brasil Giselebarbosa@poli.ufrj.br 


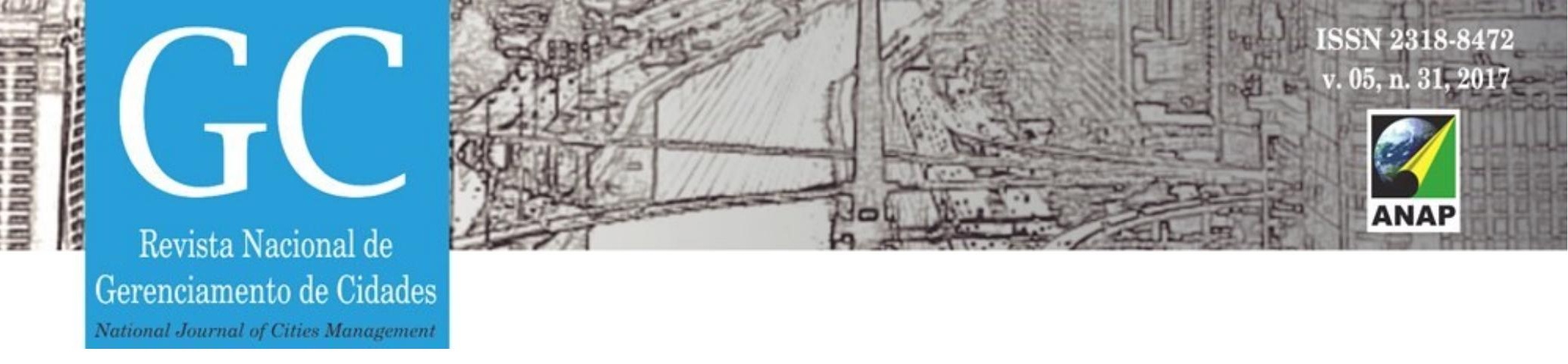

\section{RESUMO}

Diante da amplitude exacerbada das cidades, verifica-se que estas se transformaram em estruturas complexas e difíceis de administrar. Ressalta-se que as cidades deveriam existir em primeiro lugar, e acima de tudo, para satisfazer as necessidades humanas e sociais das comunidades. Apresenta-se o conceito de cidades compactas, benefícios e problemas. Neste sentido, a compactação de um lado contribui para a melhoria da qualidade de vida das pessoas, por outro, em termos energéticos, também pode influenciar negativamente nesta. Este artigo tem como objetivo propor uma solução energeticamente sustentável para a não redução da eficiência energética em modelos de cidades compactas, com foco em energia solar fotovoltaica. Através de estudos bibliográficos, verificouse que a energia solar, através de painéis fotovoltaicos é uma alternativa promissora para a redução de ilhas de calor e manutenção do conforto térmico em cidades compactas. Esta é um fator positivo para a manutenção da eficiência energética do local, respeitando-se a capacidade de suporte deste.

PALAVRAS-CHAVE: Compactação de cidade. Capacidade de suporte. Eficiência energética.

\section{ABSTRACT}

Faced with the exacerbated amplitude of the cities, these have turned into complex structures and difficult to administer. It should be stressed that cities should exist first and above all to meet the human and social needs of communities.This paper presents the concept of compact cities, benefits and problems. In this sense, the compaction of one side contributes to the improvement of the quality of life of people, on the other, in energy terms, can also negatively influence this. This article aims to propose an energetically sustainable solution for the non-reduction of energy efficiency in compact city models, focusing on photovoltaic solar energy. Through bibliographic studies, it has been verified that solar energy, through photovoltaic panels is a promising alternative for the reduction of heat islands and maintenance of thermal comfort in compact cities. This is a positive factor for the maintenance of the energy efficiency of the site, respecting its capacity of support.

KEYWORDS: City compaction. Supportability. Energy efficiency.

\section{RESUMEN}

Ante la amplitud exacerbada de las ciudades, se verifica que estas se transformaron en estructuras complejas y difíciles de administrar. Se resalta que las ciudades deberían existir en primer lugar, y sobre todo, para satisfacer las necesidades humanas y sociales de las comunidades. Se presenta el concepto de ciudades compactas, beneficios y problemas. En este sentido, la compactación de un lado contribuye a la mejora de la calidad de vida de las personas, por otro, en términos energéticos, también puede influir negativamente en ésta. Este artículo tiene como objetivo proponer una solución energéticamente sostenible para la no reducción de la eficiencia energética en modelos de ciudades compactas, con foco en energía solar fotovoltaica. A través de estudios bibliográficos, se verificó que la energía solar, a través de paneles fotovoltaicos, es una alternativa prometedora para la reducción de islas de calor y mantenimiento del confort térmico en ciudades compactas. Este es un factor positivo para el mantenimiento de la eficiencia energética del local, respetando la capacidad de soporte de éste.

PALABRAS CLAVE: Compactación de ciudad. Capacidad de soporte. Eficiencia energetica. 


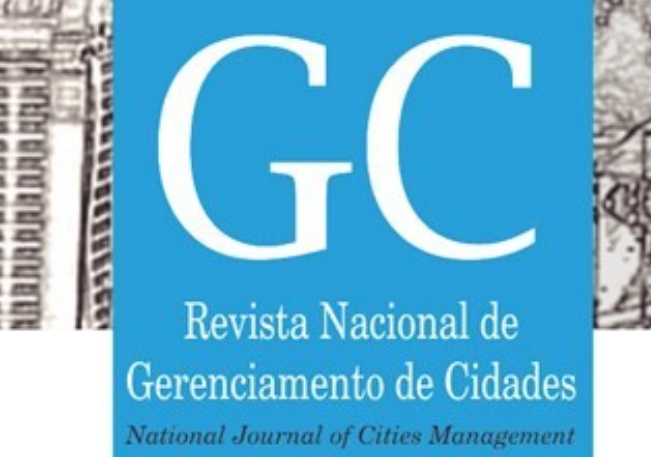

\section{INTRODUÇÃO}

As cidades cresceram e transformaram-se em estruturas tão complexas e difíceis de administrar, que por vezes, esquece-se que elas existiam em primeiro lugar, e acima de tudo, para satisfazer as necessidades humanas e sociais das comunidades. As cidades foram originalmente criadas para celebrar o que temos em comum. Atualmente, são projetadas para manter as pessoas afastados umas das outras. O crescimento urbano toma um dimensionamento capaz de comprometer a capacidade de suporte da própria cidade.

A compactação da cidade tem como maior ferramenta a manipulação do uso do solo, especialmente a uma escala urbana e regional, mas também se aplica a escalas locais, através de estratégias como o design passivo dos edifícios (desenho com preocupações de conservação energética e de outros recursos). Essa estruturação compacta tem impacto direto sobre as redes de infra-estrutura, a composição de atividades sobrepostas, permite maior convivência e reduz as necessidades de deslocamentos em automóveis, reduzindo drasticamente a energia utilizada para transporte, reduzindo também o congestionamento e melhorando a qualidade do ar, fato que estimula o cidadão a caminhar ou andar de bicicleta em substituição ao carro por exemplo.

Salienta-se que ao mesmo tempo que a compactação contribui para a qualidade de vida da população, também pode ser vista como algo limitante a esta. De fato, a compactação é capaz de influenciar negativamente na qualidade de vida dos indivíduos, trazendo-lhes desconto térmico em função da arquitetura de edifícios em determinado espaço. Os principais problemas encontrados pelo esgotamento da capacidade de suporte do ambiente são a formação de ilhas de calor e a redução da eficiência energética do local. Neste sentido, a energia solar apresenta-se como algo favorável à sustentabilidade energética para amenizar estes problemas correlatos.

Este artigo tem como objetivo propor soluções energeticamente sustentáveis para a não redução da eficiência energética em modelos de cidades compactas, com foco em energia solar fotovoltaica.

Para a elaboração deste, foi levantado estudos bibliográficos para identificar os principais problemas gerados pelo modelo compacto de cidades, com relação à qualidade de vida dos indivíduos que a habitam. Do mesmo modo, foi realizada uma análise da contribuição da energia solar para a solução de tais problemas, formalizando uma proposta de melhorias energéticas.

\section{CIDADES COMPACTAS}

A teoria de cidade compacta surge primariamente como uma tentativa de responder aos problemas internos da cidade, assim como aos problemas de sustentabilidade inerentes ao desenvolvimento das áreas urbanas. Compreender os vários modelos de formas urbanas é fundamental para entender o conceito de cidade compacta. Segundo Gomes (2009), na 


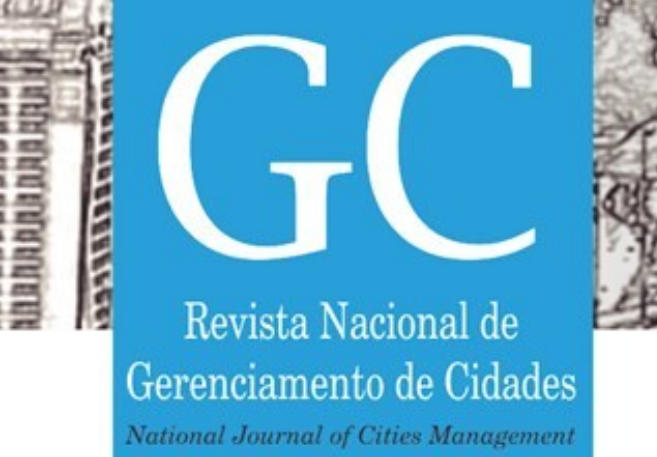

procura da forma ideal muitas vezes a teoria da cidade compacta induz a forma monocêntrica, apenas com um centro. No entanto esta forma já não corresponde às necessidades atuais da população, assim como não é sustentável e viável a partir de um determinado tamanho. Neste sentido introduz-se o conceito de cidade compacta e descentralizada.

Para Rogers (2001, apud MESTRINER 2008), uma cidade mais densa e pouco espraiada evitaria a invasão das áreas rurais, ou, como no caso de São Paulo - a não invasão das reservas ambientais. Isto traz benefícios ecológicos maiores. Através de um planejamento integrado, as cidades podem ser pensadas tendo em vista um aumento de sua eficiência energética, menor consumo de recursos, menor nível de poluição. Uma cidade densa e socialmente diversificada onde as atividades econômicas e sociais se sobreponham e onde as comunidades sejam concentradas em torno das unidades de vizinhança

A concepção de cidade compacta, segundo Mestriner (2008), prediz que o encontro das funções sociais dos cidadãos (moradia, trabalho e lazer) deve ser expresso na condição urbana que o centro propicia. Desta forma, os usos mistos e as densidades tradicionais dos centros urbanos devem trazer de volta a vivência que foi perdida após a implementação do uso do carro como transporte individual, e que, em algumas sociedades, onde essa mentalidade pobre é tão exacerbada, que o carro acaba se transformando em status social, para demonstração do poder de compra de cada indivíduo, mostrando claramente, a falta de cidadania e do espírito de coletividade necessários para se viver em comunidade. Esta ideia é representada pelo esquema da Figura 1.

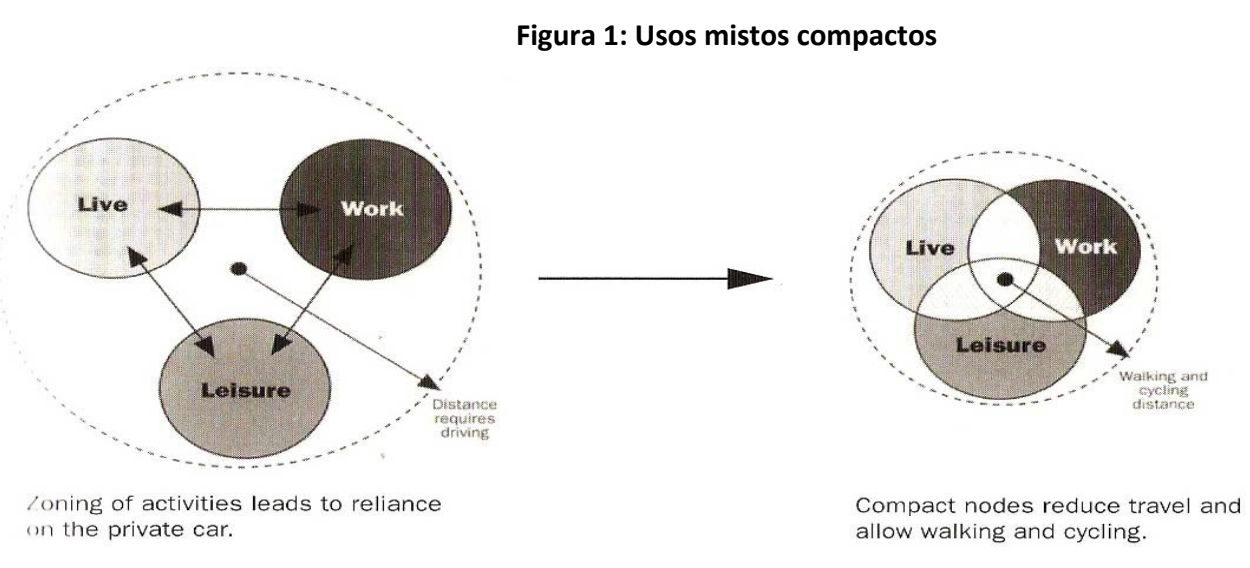

Fonte: Rogers, 2001 apud Mestriner, 2008.

Neste conceito as cidades garantem estrutura física para oportunidades de emprego e riqueza, além de habitação de qualidade para a formação de uma comunidade urbana.

O conceito de cidade compacta tem como base duas grandes características: densidades elevadas e uso de solo diversificado. Estas duas características traduzem-se numa intensificação de usos do solo, de população e de tráfego. Deste modo a cidade procura 


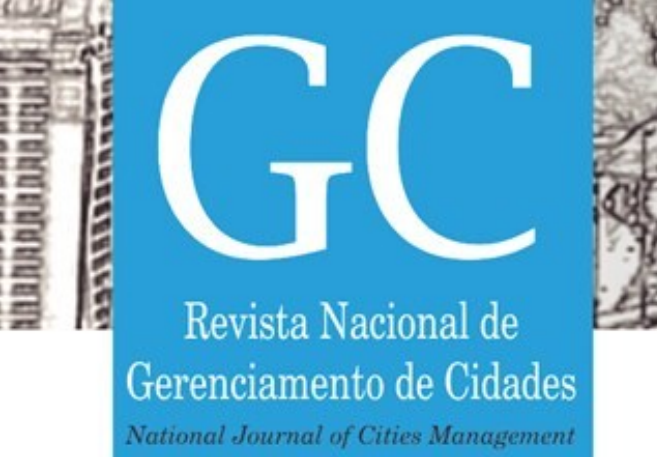

desenvolvimento, será prejudicial. A seguir são definidas as características básicas da capacidade de suporte:

- A capacidade de suporte não é o nível após o qual os impactos surgem;

- O ponto de capacidade de suporte não é o limite de saturação;

- O ponto de capacidade de suporte pode ser visto de forma diferente e conflitante por diferentes grupos;

- A capacidade de suporte incorpora dois elementos significativos: o meio ambiente e a percepção de qualidade de vida dos habitantes do lugar;

- Os aspectos da capacidade de carga a serem considerados variam de acordo com as características dos ocupantes.

O Esquema 1, enfatiza melhor o conceito de capacidade de suporte:

Esquema 1: Definição de Capacidade de suporte

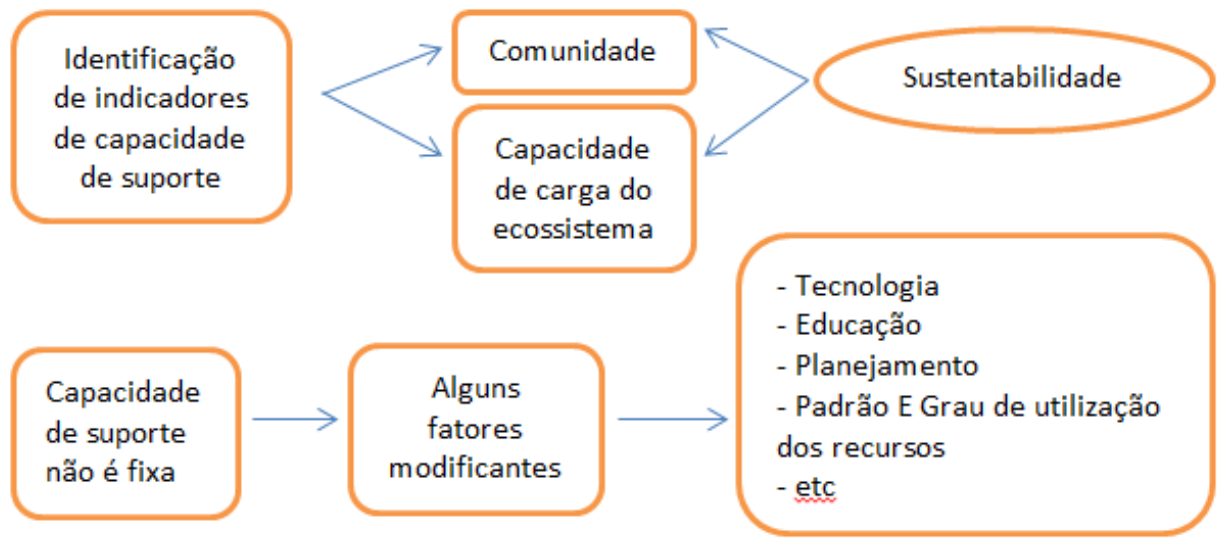

Fonte: Dias s/d apud Filet, 1955.

\section{DISCUSSÕES}

Além da valorização de áreas verdes como solução para a redução de ilhas de calor e aumento da climatização em cidades compactas, o aproveitamento de energia solar fotovoltaico pode ser uma solução promissora. A energia fotovoltaica está entre as alternativas para a implantação de microssistemas de geração de energia elétrica, pois apresenta uma maior facilidade de instalação além de apresentar operação e manutenção mais simplificada.

Fazer uso da energia elétrica convencional para resolver o problema das ilhas de calor é prejudicial ao meio ambiente. No Brasil, considerando apenas a necessidade de atender uma demanda, segundo Goldemberg \& Lucon (2007), há algumas décadas, as emissões do setor energético brasileiro não eram muito significativas dadas às características predominantemente hidrelétricas da matriz energética de energia. Mas, a partir da década de 1970, com o aumento da demanda de energia no país, foi necessária a entrada de 


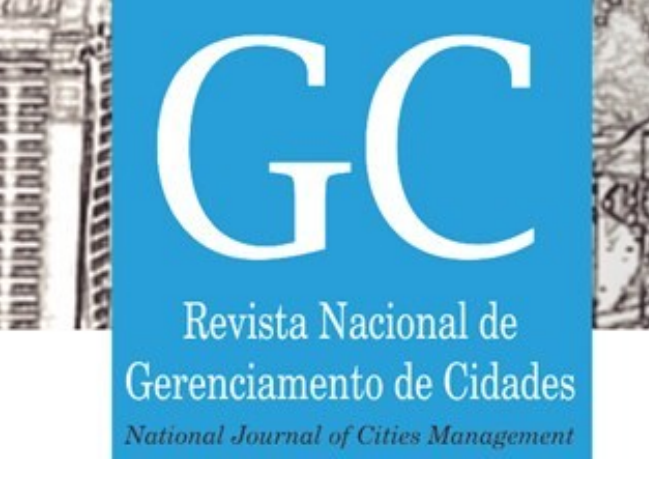

Figura 2: Forma de conexão do Acessante (através de inversor) à rede de BT da Light SESA.

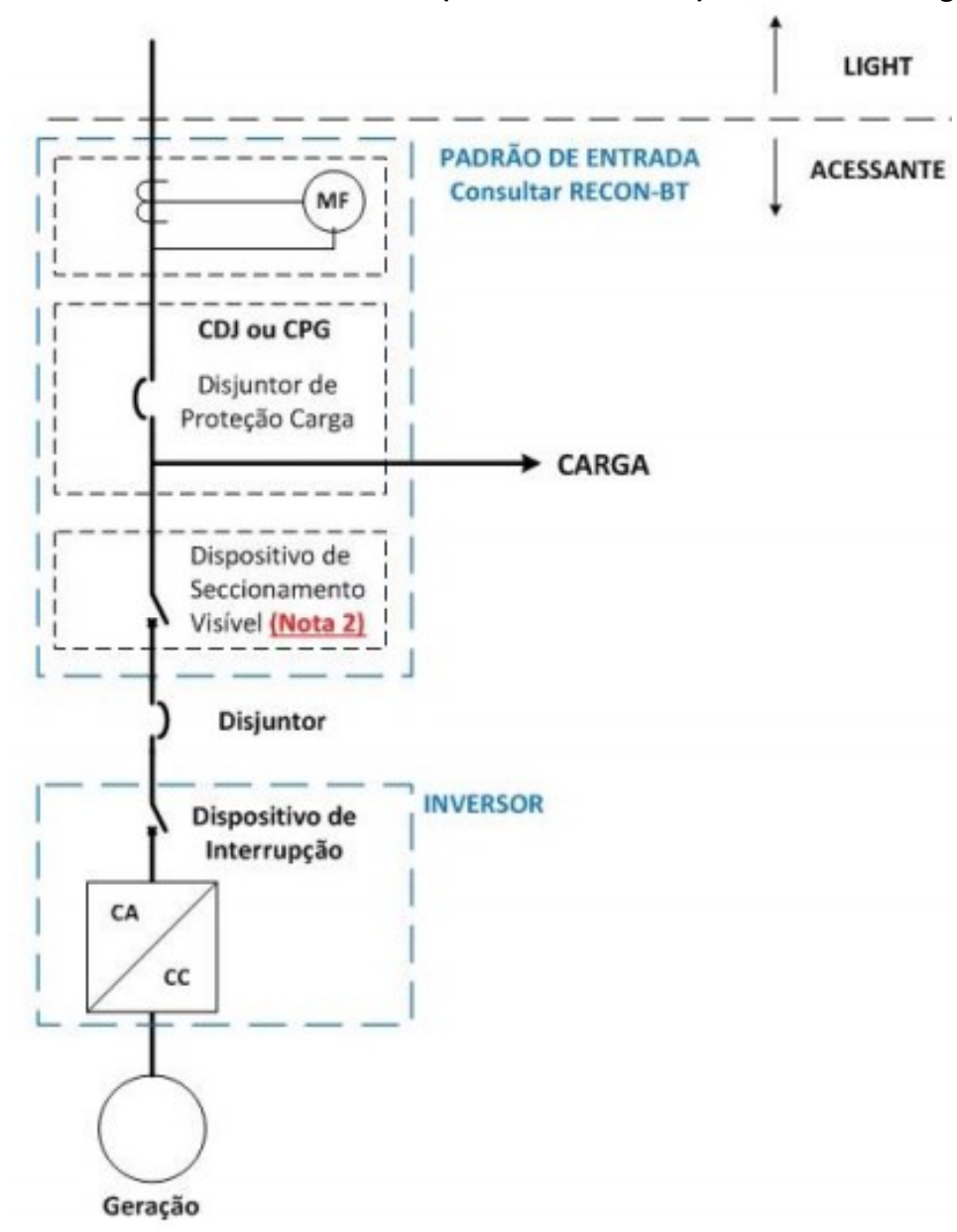

Fonte: LIGHT, 2016.

\section{CONSIDERAÇÕES FINAIS}

A teoria de cidade compacta, ao procurar por um lado diminuir as distâncias entre a origem e o destino das viagens, e por outro proporcionar um sistema de transportes públicos eficaz e de alta capacidade, potenciará uma menor dependência do automóvel, o que condicionará menor consumo de energia e menos poluição atmosférica. Para além da redução da dependência do automóvel, são facilitados: o aumento da acessibilidade, o rejuvenescimento das áreas centrais, a preocupação na manutenção e qualidade dos espaços verdes e a qualidade dos espaços públicos. Estes aspectos aumentam a qualidade de vida global das 
